\title{
Características tomográficas de la bifurcación del conducto dentario inferior
}

\author{
Tomographic characteristics of the bifurcation of the mandibular canal \\ Magaly Lizbet Quispe-Huarcaya ${ }^{1, a}$, Milushka Miroslava Quezada-Márquez ${ }^{1, b, c, d}$, Roberto Antonio León-Manco ${ }^{2, e}$
}

\section{RESUMEN}

Objetivos: Evaluar las características tomográficas de la bifurcación del conducto dentario inferior (BCDI) mediante tomografía computarizada de haz cónico (TCHC). Material y Métodos: Se revisaron 1497 TCHC (527 hombres y 970 mujeres) del servicio de Radiología Oral y Maxilofacial de la Facultad de Estomatología de la Universidad Peruana Cayetano Heredia, periodo 2011 - 2014. El análisis de imágenes se realizó utilizando el software Galileos 1.7.2. El rango etario estuvo comprendido entre 10 a 89 años, con una media de $44.8 \pm 14$ años. Asimismo se utilizó la clasificación de Naitoh, Hiraiwa, Aimiya y Ariji. El análisis estadístico se realizó mediante el uso de la prueba chi-cuadrado. Resultados: La frecuencia de la BCDI fue del 10.75\% (161 casos). Se encontró mayor frecuencia en la quinta década de vida para ambos sexos, no hubo diferencia estadísticamente significativa entre el sexo femenino y masculino ( $\mathrm{p}=0.49$ ). La distribución de la BCDI evidenció que el tipo Canal retromolar representó el 64\% (103 casos), seguido del tipo Canal sin confluencia anterior con 21.1\% (34 casos) y minoritariamente el Canal dental y Canal con confluencia anterior con un 9.9\% (16 casos) y 5\% (8 casos) respectivamente. Conclusiones: La BCDI tiene una prevalencia del 10.75\% y la TCHC permite identificar oportunamente esta variante anatómica.

PALABRAS CLAVE: Variación anatómica, tomografía computarizada de haz cónico, canal mandibular bífido, nervio dentario inferior.

\section{SUMMARY}

Objectives: To evaluate the tomographic characteristics of the bifurcation of the mandibular canal (BCDI) using cone beam computed tomography (CBCT). Material and Methods: 1497 CBCT were reviewed (from 527 men and 970 women), of the Department of Oral and Maxillofacial Radiology, from Universidad Peruana Cayetano Heredia, period 2011 - 2014. Image analysis were made using the software 1.7.2 Galilee. Patients age was between 10 to 89 years old, averaging $44.8 \pm 14$ years. Naitoh classification, Hiraiwa, Aimiya and Ariji was also

\footnotetext{
${ }^{1}$ Facultad de Estomatología, Universidad Peruana Cayetano Heredia. Lima, Perú.

2 Departamento Académico de Odontología Social, Facultad de Estomatología, Universidad Peruana Cayetano Heredia. Lima, Perú.

a Residente de la Especialidad de Radiología Bucal y Maxilofacial.

b Docente.

c Especialista en Radiología Oral y Maxilofacial.

d Magister en Estomatología con mención en Radiología Oral y Maxilofacial.

e Docente auxiliar
} 
used. Statistical analysis was made by using the chi-square test. Results: The frequency of BCDI was 10.75\% (161 cases). The highest frequency was found in the fifth decade of life for both sexes, there wasn't statistically significant difference between female and male sex $(\mathrm{p}=0.49)$. The distribution of the BCDI showed that the kind Canal retromolar represents 64\% (103 cases), followed by the type Canal without previous confluence with 21.1\% (34 cases) and minority was found the dental Canal and Canal above confluence with 9.9\% (16 cases) and 5\% (8 cases) respectively. Conclusions: The bifurcation of mandibular canal has a prevalence of 10.75\% and CBCT allows timely identification of this anatomical variant.

\section{KEYWORDS: Anatomical variation, cone beam computed tomography, bifid mandibular canal, inferior alveolar nerve.}

\section{INTRODUCCIÓN}

La evolución en el campo de la radiología ha permitido visualizar hasta las partes más pequeñas del cuerpo humano, por ello su papel es fundamental en la detección de las enfermedades. La tomografía computarizada de haz cónico permite obtener imágenes en los tres planos del espacio mejorando la visualización de las estructuras anatómicas del macizo facial y principalmente de las variantes que éstas puedan presentar. El conducto dentario inferior está ubicado en la mandíbula por donde recorren los nervios y vasos alveolares inferiores; dicho canal nace en la cara interna de la rama ascendente por detrás de la espina de Spix o língula, desde donde se dirige hacia abajo y adelante generalmente hasta el segundo premolar donde se divide en dos ramas: una rama externa (conducto mentoniano) que termina en el foramen mentoniano y otra rama interna (conducto incisivo) que termina debajo de los incisivos antero inferiores. Al examinarlo por secciones puede presentarse con diferentes formas: circular, oval y piriforme (1). Las investigaciones coinciden en que el conducto dentario inferior se encuentra en mayor porcentaje más cercano a la tabla ósea lingual y al margen inferior de la mandíbula (2). El nervio dentario inferior es la rama más voluminosa del tronco posterior del nervio mandibular que, a su vez, es la tercera rama del nervio trigémino $(1,2)$. Es formado durante el desarrollo embrionario para inervar tres diferentes grupos dentarios, incisivos temporales, molares temporales y dientes permanentes, proceso que termina con la fusión de estos tres nervios en uno, el nervio dentario inferior. Se piensa que probablemente la permanencia o fusión incompleta de estos nervios daría origen a los conductos mandibulares bífidos (4).

La bifurcación del conducto dentario inferior (BCDI) es una variante anatómica del canal mandibular donde anatómicamente se observa dicho conducto divi- dido en dos ramas, cada una separada en su propio canal por donde discurre una rama neurovascular que puede ser observado en diferentes formas; puede presentarse tanto en un solo lado de la rama mandibular como en ambos lados (5). Un estudio realizado por el investigador Fukami et al., (6) observaron la presencia de conductos mandibulares bífidos en cadáveres por medio de tomografía computarizada volumétrica y realizó cortes histológicos evidenciando que dichos conductos contenían haces de nervios y arterias de diversos tamaños.

Reconocer la ubicación y configuración del conducto dentario inferior es importante en cualquier tipo de cirugía bucal que implique procedimientos en la mandíbula, tales como la extracción del tercer molar, implante dental y osteotomía sagital de rama. Existen muchos casos de fracaso en el bloqueo del nervio dentario inferior por la presencia del conducto mandibular bífido (7).

Existen numerosos informes sobre la frecuencia de la bifurcación del conducto dentario inferior. La ocurrencia de la bifurcación del conducto dentario inferior ha sido informada en 0.35\% según Sanchis et al., (8) (España), $0.9 \%$ por Nortje et al., (9) (Sudáfrica) y $0.95 \%$ por Langlais et al., (10) (EE.UU). Klinge et al., reportaron que el canal mandibular en muestras de cadáveres no es visible en el 36.1\% de las radiografías panorámicas (11.12). En estudios más recientes realizados mediante tomografia computarizada de haz cónicoTCHC la incidencia de BCDI ha sido reportada entre el 15.6\% - 64.8\% (13-17). El rango de diferencia en los resultados por medio de las dos técnicas es muy alto, esto se debe a las limitaciones que presenta la radiografía panorámica convencional, ya que observamos estructuras tridimensionales en una imagen de dos dimensiones (18-20).

En la literatura existen varias clasificaciones para definir los tipos que se presentan en la BCDI, se han descrito y clasificado de acuerdo con la localización y 


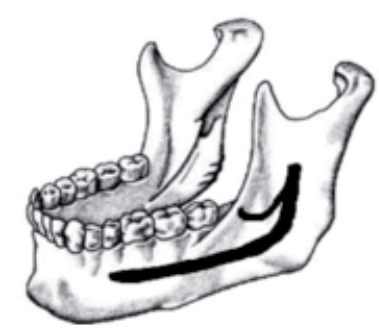

A

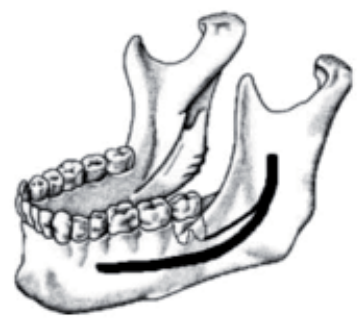

B
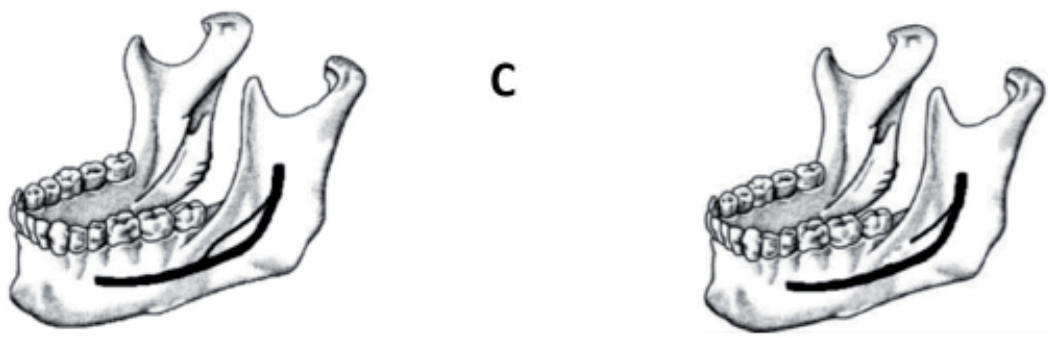

D

$\mathbf{E}$

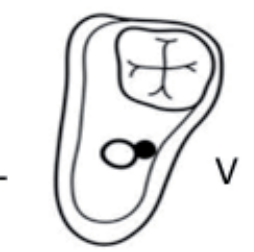

$\mathbf{F}$

$\mathrm{L}$

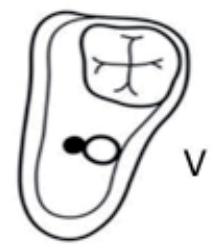

C

Figura 1. Gráfico de la clasificación de Naitoh, Hiraiwa, Aimiya y Ariji

configuración anatómica. Claeys y Wacken nombran en su revisión bibliográfica las clasificaciones realizadas por medio de radiografía panorámica (5), las cuales incluyen las clasificaciones de los investigadores Carter y Keen en el año 1971 (3), la investigación de Nortje et al., realizada en 1977 (9) y el estudio de Langlais et al., en 1985 (10).

Los investigadores Naitoh et al., en el año 2009 contribuyen con la clasificación más reciente del conducto mandibular bífido además de ser la primera clasificación realizada mediante TCHC donde consideraron 4 tipos (Figura 1) (15).

Tipo 1 (Canal retromolar): Consiste en una bifurcación del canal mandibular en la región de la rama mandibular, no alcanza piezas dentales, el conducto realiza una curva y alcanza la región retromolar (Figura $1(\mathrm{~A})$ ).

Tipo 2 (Canal dental): En este tipo la bifurcación del canal mandibular alcanza el ápice de la raíz del segundo o tercer molar (Figura 1(B)).

Tipo 3 (Con y sin confluencia anterior): Bifurcación del conducto mandibular, el cual continúa su recorrido hacia el sector anterior, luego puede volver a unirse o no con el conducto mandibular principal (Figura
1 (C y D)).

Tipo 4 (Canal bucal - lingual): Bifurcación del conducto mandibular, el cual recorre por el sector lingual o bucal del cuerpo de la mandíbula y del canal mandibular principal (Figura 1 (E y F))

La identificación de la situación del conducto dentario inferior así como el conocimiento de cualquier variante anatómica es importante debido al compromiso clínico que se pueda producir, como es el fallo en la técnica anestésica, neuropatías iatrogenias y otras situaciones a tener en cuenta durante los procedimientos quirúrgicos que abarquen esta zona anatómica (9.14). Cuando la cirugía del tercer molar ha de llevarse a cabo, se debe tener precaución con ésta variante anatómica en la zona molar. El diente puede infringir o estar dentro del canal en sí. Un segundo paquete neurovascular puede estar contenido en los canales bífidos, complicaciones como neuroma traumático, parestesia y sangrado podrían surgir a causa de falta del reconocimiento de esta anomalía (16.17).

La detección de la BCDI se facilitó a partir de 1973 con la introducción del tomógrafo axial computari- 
zada por Hounsfield y Cormack, de amplio uso en el campo médico. Fue a partir de 1999, con la introducción de la TCHC que los radiólogos maxilofaciales cuentan con un equipo específico para el área de su interés, evidenciando la alta frecuencia de conductos mandibulares bífidos (19.20).

\section{MATERIAL Y MÉTODOS}

El diseño del presente estudio fue de tipo descriptivo, transversal y retrospectivo. La selección de las TCHC se realizó a través de un muestreo por conveniencia utilizando las tomografías que cumplían con los criterios de inclusión, tomadas del Servicio de Radiología Oral y Maxilofacial del Posgrado de la Facultad de Estomatología de la Universidad Peruana Cayetano Heredia (UPCH), periodo 2011-2014. Se incluyeron las TCHC nítidas de pacientes de ambos sexos. Se excluyeron aquellas tomografías de pacientes con aparatología ortodóntica, patologías tumorales y quísticas, así como fracturas que comprometían la zona de estudio y/o malformaciones craneofaciales. Las variables tomadas en cuenta fueron: tipo de bifurcación, localización, edad y sexo.

La investigadora principal fue capacitada y calibrada por un radiólogo oral y maxilofacial con una experiencia mayor a 10 años. Dicha calibración fue desarrollada en 15 tomografías computarizadas de haz cónico (Siemens, Alemania) que opera de $10 \mathrm{~mA}$ a $42 \mathrm{~mA}$ y $85 \mathrm{kV}$ de acuerdo a cada paciente, todas las imágenes digitales fueron exportadas a una computadora Lenovo H61 y evaluadas a través del software Galileos 1.7.2. El valor de Kappa interobservador fue de 0,86 y el Kappa intraobservador fue de 0.89. Se evaluó diez TCHC por día y de forma interdiaria. Todas las sesiones de visualización se llevaron a cabo en una habitación tranquila con luz ambiental tenue. Las imágenes digitales se analizaron en una pantalla de alta resolución de 21 pulgadas, la resolución de pantalla se fijó en 1600x900 píxeles con una profundidad de color de 32 bits. Se usó distribución de frecuencia para las variables de sexo, localización y tipo de bifurcación. Así mismo se realizó la prueba estadística Chi cuadrado y Chi cuadrado corregido por Yates para las variables sexo y tipo de bifurcación. El estudio contó con un nivel de confianza de 95\% y $\mathrm{p}<0.05$. Los análisis fueron realizados en el programa estadístico 23.0.

\section{RESULTADOS}

Se revisaron 1497 tomografías de los cuales $10.75 \%$ (161 casos) presentaron bifurcación del conducto dentario inferior. En la población el género femenino fue el 64.8\% (970 casos) y el género masculino fue el $35.2 \%$ (527 casos). La prevalencia de la BCDI en el género femenino fue de $11.03 \%$ (107 casos) y en el masculino fue de $10.2 \%$ (54 casos) (Tabla 1).

La edad de la población fue de un rango etario de 10 a 89 años, con una media de 44,8 \pm 14 años. La BCDI se encontró en su mayoría en la quinta década de vida con un $26.7 \%$ (43 casos) y en su minoría en la segunda y novena década con un 1.2\% (2 casos) (Tabla 2).

En la tabla 3 se describe la distribución de la frecuencia del tipo de BCDI donde se encontraron 103 casos del tipo canal retromolar (Figuras 1, 2 y 3); no se encontró ningún caso con el tipo de bifurcación canal bucal-lingual.

Del total de casos encontrados con BCDI el 93.79\% (151 casos) fueron unilaterales y 6.21\% (10 casos) bilaterales (Tabla 4).

En la tabla 5 se muestra que no hubo diferencia estadísticamente significativa entre ambos lados $(p=0.78)$ (Figuras 5 y 6 ).

No hubo diferencia estadísticamente significativa entre el género femenino y masculino ( $\mathrm{p}=0.49$ ) (Tabla 6).

Tabla 1. Distribución del sexo del total de la población y de los pacientes con BCDI evaluados con TCHC.

\begin{tabular}{lccc}
\hline \multicolumn{1}{c}{ Género } & Total de Población & Muestra con Conducto Bífido & Porcentaje (\%) \\
\hline Masculino & 527 & 54 & 10.25 \\
Femenino & 970 & 107 & 11.03 \\
TOTAL & 1497 & 161 & 10.75 \\
\hline
\end{tabular}

Prueba de Chi cuadrado $\mathrm{p}=0,6418$ 
Tabla 2. Distribución de la BCDI según grupo etario evaluados con TCHC.

\begin{tabular}{lcccccc}
\hline \multicolumn{1}{c}{ GRUPO ETARIO } & \multicolumn{2}{c}{ Masculino } & \multicolumn{2}{c}{ Femenino } & \multicolumn{2}{c}{ TOTAL } \\
& $\mathbf{n}$ & $\mathbf{\%}$ & $\mathbf{n}$ & $\mathbf{\%}$ & $\mathbf{~ N}$ & $\mathbf{\%}$ \\
\hline 10 a 19 años & 1 & 0.6 & 1 & 0.6 & 2 & 1.2 \\
20 a 29 años & 12 & 7.5 & 14 & 8.7 & 26 & 16.2 \\
30 a 39 años & 6 & 3.7 & 24 & 14.9 & 30 & 18.6 \\
40 a 49 años & 16 & 9.9 & 27 & 16.8 & 43 & 26.7 \\
50 a 59 años & 9 & 5.6 & 23 & 14.3 & 32 & 19.9 \\
60 a 69 años & 8 & 5 & 15 & 9.3 & 23 & 14.3 \\
70 a 79 años & 1 & 0.6 & 2 & 1.3 & 3 & 1.9 \\
80 a 89 años & 1 & 0.6 & 1 & 0.6 & 2 & 1.2 \\
TOTAL & 54 & 33.5 & 107 & 66.5 & 161 & 100 \\
\hline
\end{tabular}

Tabla 3. Distribución de la frecuencia de los tipos* de BCDI según clasificación de Naitoh et al.

\begin{tabular}{lcc}
\hline TIPO DE BIFURCACIÓN* & $\mathbf{n}$ & $\mathbf{\%}$ \\
Canal Retromolar & 103 & 64.0 \\
Canal Dental & 16 & 9.9 \\
Con Confluencia Anterior & 8 & 5.0 \\
Sin Confluencia Anterior & 34 & 21.1 \\
TOTAL & 161 & 100.0 \\
\hline
\end{tabular}

*Clasificación de Naitoh, Hiraiwa, Aimiya y Ariji (21)

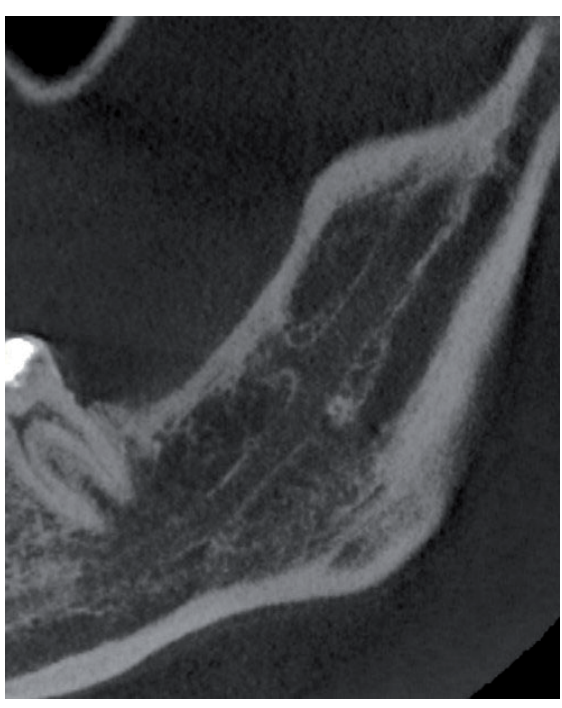

Figura 2. Canal dentario bífido tipo retromolar izquierdo.

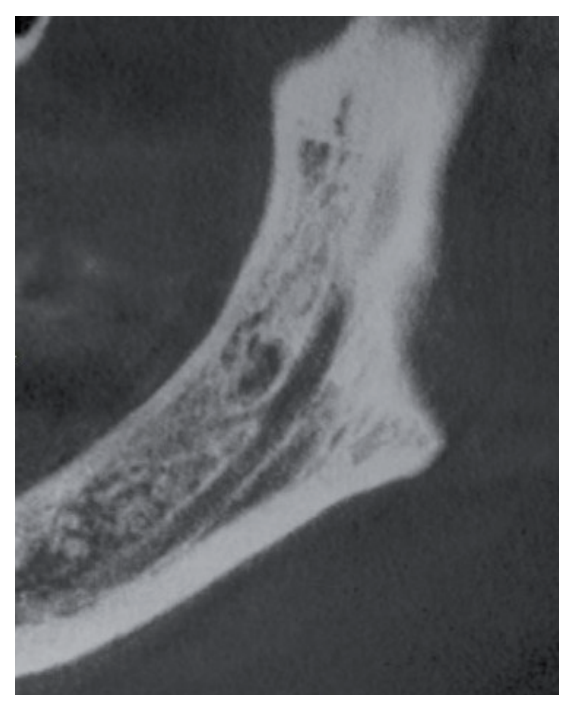

Figura 3. Bifurcación del conducto dentario inferior. Tipo retromolar con emergencia en la zona del tercer molar.

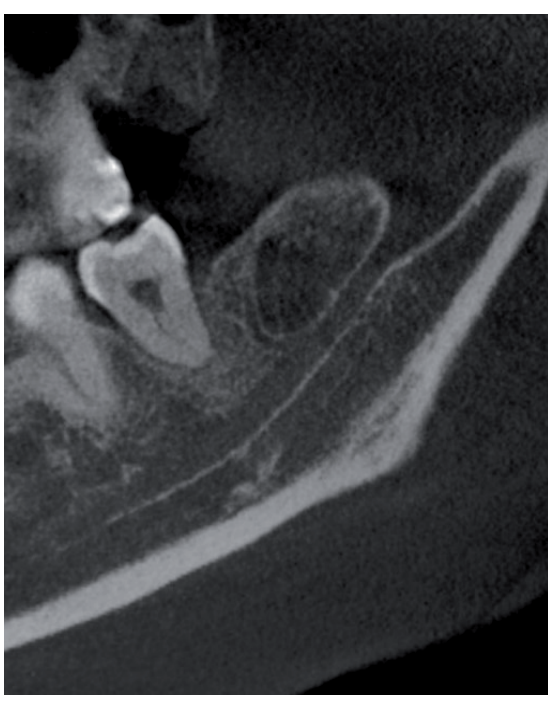

Figura 4. Bifurcación del conducto dentario inferior. Tipo retromolar emergiendo en la zona distal del tercer molar. 
Tabla 4. Distribución de la frecuencia de los tipos* de BCDI según localización (bilateralidad).

\begin{tabular}{lcccc}
\hline \multicolumn{1}{c}{ TIPO DE BIFURCACIÓN* } & \multicolumn{2}{c}{ LOCALIZACIÓN } \\
& Unilateral & \multicolumn{2}{c}{ Bilateral } \\
\hline Canal retromolar & $\mathbf{n}$ & $\mathbf{\%}$ & $\mathbf{n}$ & $\mathbf{\%}$ \\
Canal dental & 95 & 59.01 & 8 & 4.97 \\
Con confluencia anterior & 16 & 9.94 & 0 & 0.0 \\
Sin confluencia anterior & 7 & 4.35 & 1 & 0.62 \\
TOTAL & 33 & 20.50 & 1 & 0.62 \\
\hline *Clasificación de Naitoh, Hiraiwa, Aimiya y Ariji ${ }^{21}$ & 151 & 93.79 & 10 & 6.21 \\
\hline
\end{tabular}

Tabla 5. Distribución de la frecuencia de los tipos* de BCDI según localización (lado).

\begin{tabular}{lcccc}
\hline \multicolumn{1}{c}{ TIPO DE BIFURCACIÓN* } & \multicolumn{3}{c}{ LOCALIZACIÓN } \\
& Derecho & \multicolumn{2}{c}{ Izquierdo } \\
\hline Canal retromolar & $\mathbf{n}$ & $\mathbf{\%}$ & $\mathbf{n}$ & $\mathbf{\%}$ \\
Canal dental & 45 & 29.80 & 50 & 33.11 \\
Con confluencia anterior & 12 & 7.95 & 4 & 2.65 \\
Sin confluencia anterior & 4 & 2.65 & 3 & 1.99 \\
TOTAL & 16 & 10.65 & 17 & 11.26 \\
\hline *Clasificación de Naitoh, Hiraiwa, Aimiya y Ariji ${ }^{21}$ & 77 & 50.99 & 74 & 49.01 \\
\hline
\end{tabular}

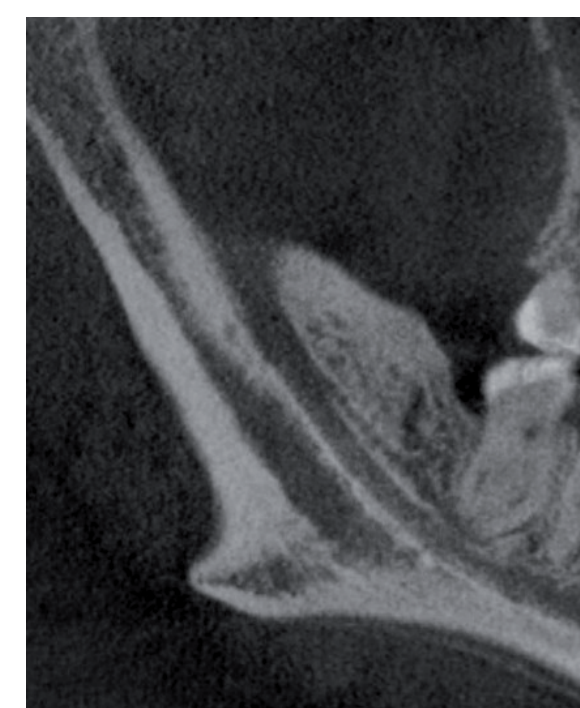

Figura 5. Bifurcación del conducto dentario inferior. Tipo canal sin confluencia anterior.

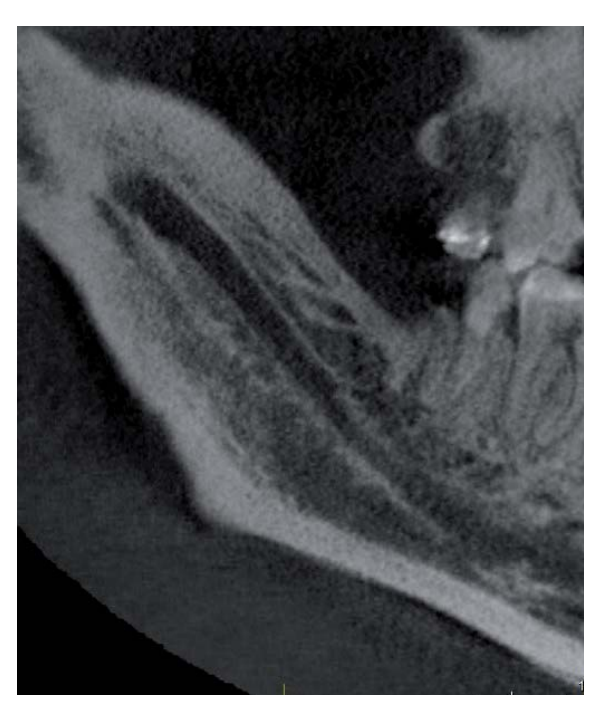

Figura 6. Bifurcación del conducto dentario inferior. Tipo canal dental. 
Tabla 6. Distribución de la frecuencia del tipo de BCDI según género.

\begin{tabular}{lcccc}
\hline \multicolumn{1}{c}{ TIPO DE BIFURCACIÓN } & \multicolumn{2}{c}{ GÉNERO } \\
& \multicolumn{2}{c}{ Masculino } & \multicolumn{2}{c}{ Femenino } \\
& $\mathbf{n}$ & $\mathbf{\%}$ & $\mathbf{n}$ & $\mathbf{\%}$ \\
\hline Canal Retromolar & 33 & 20.50 & 70 & 43.48 \\
Canal Dental & 4 & 2.48 & 12 & 7.45 \\
Con Confluencia Anterior & 5 & 3.11 & 3 & 1.86 \\
Sin Confluencia Anterior & 12 & 7.45 & 22 & 13.66 \\
TOTAL & 54 & 33.54 & 107 & 66.46 \\
\hline
\end{tabular}

Chi cuadrado corregido por Yates $(\mathrm{p}=0.49)$

\section{DISCUSIÓN}

La bifurcación del conducto dentario inferior constituye un hallazgo imaginológico de gran importancia que está presente en una pequeña parte de la población; dicha variante anatómica pasa desapercibida a pesar de que se pueden registrar en las radiografías panorámicas; sin embargo la TCHC es considerada el método de elección para su estudio ya que brinda mejor información sobre el curso exacto del canal mandibular.

El canal mandibular bífido, particularmente el tipo canal retromolar, tiene implicaciones clínicas importantes principalmente en cirugía de terceros molares, exodoncias de dientes posteriores en el maxilar inferior, cirugía sagital de rama (cirugía ortognática), obtención de injertos óseos en la zona retromolar, tratamientos de conductos radiculares y eventualmente como sitio de elección para la colocación de implantes oseointegrados $(12,16)$. Aunque muchos estudios citan el conducto dentario inferior como una sola estructura bilateral, hay varios informes en la literatura que demuestran claramente la presencia de un segundo o incluso tercer conducto accesorio $(13,18,20)$.

El presente estudio es uno de los pocos que evalúan esta variante anatómica con tomografía computarizada volumétrica, la gran ventaja de esta técnica radica en que nos brinda una vista tridimensional de las estructuras anatómicas, siendo posible detectar con más precisión la BCDI ya que la TCHC fue capaz de detectar estrechos canales que pueden no haber sido detectados por imágenes panorámicas $(14,17)$.
La frecuencia de la bifurcación del conducto dentario inferior en este estudio fue de $10.75 \%$ (161 casos) al revisar 1497 tomografías computarizadas volumétricas similar a otros estudios. En el estudio realizado por Naitoh et al., observaron 65\% de las bifurcaciones del canal mandibular en 122 exploraciones con TCHC (15). Por otro lado, Kuribayashi et al., en Japón observaron 15\% de los canales bifurcados en 252 tomografías (16); asimismo Muinelo et al., en Japón encontraron $36.8 \%$ de canales mandibulares bífidos en 225 pacientes examinados con TCHC y un 16.8\% mediante el uso de radiografía panorámica (18). Rashsuren et al., en España al revisar 500 tomografías encontraron un $22.6 \%$ de prevalencia de esta variante anatómica (17). En Sudamérica el estudio realizado por Videla et al., en Chile, de un total de 84 pacientes examinados con TCHC encontraron que el 69\% (54 casos) presentó BCDI (14). Sin embargo la alta prevalencia de esta variante anatómica encontrada por Videla está en relación a la menor población evaluada. En nuestro estudio se evaluó una mayor población (1497 tomografías volumétricas) encontrando una prevalencia de $10.75 \%$ (Tabla 7 ).

La frecuencia en la detección de esta variante anatómica aumenta al 64.8\% cuando se utiliza TCHC en comparación con la radiografía panorámica (1620). Está claro que con los avances en la tecnología y la mejora de las imágenes como herramientas de diagnóstico, el número de variaciones anatómicas del canal mandibular es probable que aumente considerablemente.

La tabla 8 muestra la distribución del género de pacientes con bifurcación del conducto dentario 
Tabla 7. Tabla resumen de la frecuencia de bifurcaciones del conducto dentario inferior.

\begin{tabular}{|c|c|c|c|c|c|c|}
\hline Autores & Año & País & $\begin{array}{c}\text { Total de pacientes } \\
\text { evaluados }\end{array}$ & $\begin{array}{c}\mathrm{N}^{\circ} \text { de pacientes con } \\
\text { BCDI }\end{array}$ & $\%$ & $\begin{array}{c}\text { Técnica de } \\
\text { imagen }\end{array}$ \\
\hline Quispe & 2016 & Perú & 1497 & 161 & 10.8 & TCHC \\
\hline Rashsuren et al. ${ }^{17}$ & 2014 & Korea & 500 & 113 & 22.6 & TCHC \\
\hline Muinelo et al. ${ }^{18}$ & 2012 & España & 225 & 83 & 36.8 & TCHC \\
\hline Muinelo et al. ${ }^{18}$ & 2012 & España & 225 & 38 & 16.8 & Panorámica \\
\hline Kang et al. ${ }^{19}$ & 2011 & Korea & 1933 & 198 & 10.2 & TCHC \\
\hline Videla et al. ${ }^{14}$ & 2010 & Chile & 84 & 54 & 69 & TCHC \\
\hline Naitoh et al. ${ }^{15}$ & 2009 & Japón & 122 & 79 & 65 & TCHC \\
\hline Rossi et al. ${ }^{12}$ & 2009 & Brasil & 500 & 43 & 8.6 & Panorámica \\
\hline Schilling et al. ${ }^{11}$ & 2008 & Chile & 350 & 35 & 5 & Panorámica \\
\hline Kuribayashi et al. ${ }^{16}$ & 2005 & Japón & 252 & 47 & 15.6 & TCHC \\
\hline Langlais et al. ${ }^{10}$ & 1985 & EEUU & 6000 & 57 & 0.95 & Panorámica \\
\hline Nortjé et al. ${ }^{9}$ & 1977 & Sudáfrica & 3612 & 33 & 1 & Panorámica \\
\hline
\end{tabular}

BCDI: Bifurcación del conducto dentario inferior.

TCHC: Tomografía computarizada de haz cónico.

Tabla 8. Tabla resumen del género de pacientes con bifurcación del conducto dentario inferior.

\begin{tabular}{|c|c|c|c|c|c|c|}
\hline \multirow{3}{*}{ Autores } & \multirow{3}{*}{ Año } & \multirow{3}{*}{ País } & \multicolumn{4}{|c|}{ Género } \\
\hline & & & \multicolumn{2}{|c|}{ Femenino } & \multicolumn{2}{|c|}{ Masculino } \\
\hline & & & $\mathbf{n}$ & $\%$ & $\mathbf{n}$ & $\%$ \\
\hline Quispe & 2016 & Perú & 107 & 11.03 & 54 & 10.2 \\
\hline Rashsuren et al. ${ }^{17}$ & 2014 & Korea & 52 & 24.7 & 61 & 21 \\
\hline Muinelo et al. ${ }^{18}$ & 2012 & España & 39 & 28.8 & 44 & 48.8 \\
\hline Muinelo et al. ${ }^{18}$ & 2012 & España & 18 & 14 & 20 & 21.1 \\
\hline Kang et al. ${ }^{19}$ & 2011 & Korea & 97 & $49 *$ & 101 & $51 *$ \\
\hline Videla et al. ${ }^{14}$ & 2010 & Chile & 52 & $61.91^{*}$ & 32 & $38.09 *$ \\
\hline Naitoh et al. ${ }^{15}$ & 2009 & Japón & 55 & 45 & 24 & 19.7 \\
\hline Rossi et al. ${ }^{12}$ & 2009 & Brasil & 328 & $65.6 *$ & 172 & $34.4^{*}$ \\
\hline Schilling et al. ${ }^{11}$ & 2008 & Chile & 203 & 29 & 147 & 21 \\
\hline Kuribayashi et al. ${ }^{16}$ & 2005 & Japón & 31 & 10.3 & 16 & 5.3 \\
\hline Langlais et al. ${ }^{10}$ & 1985 & EEUU & 38 & 1.03 & 19 & 0.81 \\
\hline Nortjé et al. ${ }^{9}$ & 1977 & Sudáfrica & 1099 & $50.5^{*}$ & 668 & $46.5^{*}$ \\
\hline
\end{tabular}

Porcentajes hallados con los pacientes que presentaban bifurcación del conducto dentario inferior.

* Porcentajes hallados del total de pacientes evaluados. 
inferior. En la revisión de la literatura algunos investigadores han reportado una ligera predilección por el sexo femenino para la incidencia de la BCDI mediante radiografías panorámicas teniendo en cuenta el total de pacientes evaluados,sin embargo en nuestro estudio no hubo diferencia estadísticamente significativa entre ambos sexos.

Con respecto a la edad de los pacientes con canal mandibular bífido, en presente estudio, estuvo en un rango de 10-89 años. La frecuencia de esta variante anatómica no se incrementa con la edad debido a que se forma durante la embriogénesis, por el contrario su hallazgo en edades avanzadas podría disminuir producto de las condiciones óseas propias de la edad (Tabla 9).

La tabla 10 muestra la distribución de los tipos de BCDI según la clasificación de Naitoh. Hiraiwa, Aimiya y Ariji en los estudios realizados sobre esta variante anatómica.

Tabla 9. Tabla resumen de la edad de los pacientes BCDI.

\begin{tabular}{|c|c|c|c|c|c|}
\hline Autores & Año & País & $\begin{array}{c}\text { Rango de } \\
\text { edad (años) }\end{array}$ & $\begin{array}{l}\text { Grupo etario de mayor fre- } \\
\text { cuencia BCDI (años) }\end{array}$ & $\begin{array}{l}\text { Promedio } \\
\text { (años) }\end{array}$ \\
\hline Quispe & 2016 & Perú & $10-89$ & $40-49$ & 44.5 \\
\hline Rashsuren et al. ${ }^{17}$ & 2014 & Korea & $0-50$ & $40-49$ & - \\
\hline Muinelo et al. ${ }^{18}$ & 2012 & España & $13-19$ & - & 43.87 \\
\hline Kang et al. ${ }^{19}$ & 2011 & Korea & $13-93$ & $20-29$ & 28.4 \\
\hline Videla et al. ${ }^{14}$ & 2010 & Chile & $12-80$ & - & 50.8 \\
\hline Naitoh et al. ${ }^{15}$ & 2009 & Japón & $17-78$ & - & 50.8 \\
\hline Schilling et al. ${ }^{11}$ & 2008 & Chile & $13-98$ & - & 30.4 \\
\hline Kuribayashi et al. ${ }^{16}$ & 2005 & Japón & $18-74$ & - & 33 \\
\hline Langlais et al. ${ }^{10}$ & 1985 & EEUU & $0-81$ & $31-40$ & - \\
\hline Nortjé et al. ${ }^{9}$ & 1977 & Sudáfrica & $0-60$ & $20-29$ & - \\
\hline
\end{tabular}

BCDI: Bifurcación del conducto dentario inferior.

Tabla 10: Tabla resumen de la distribución del tipo BCDI según la clasificación de Naitoh et al.

\begin{tabular}{|c|c|c|c|c|c|c|}
\hline Autores & Año & País & $\begin{array}{c}\text { Canal } \\
\text { retromolar }\end{array}$ & Canal dental & Canal anterior & $\begin{array}{c}\text { Canal } \\
\text { bucolingual }\end{array}$ \\
\hline Quispe & 2016 & Perú & 64 & 9.9 & 26.1 & 0 \\
\hline Rashsuren et al. ${ }^{17}$ & 2014 & Korea & 71.3 & 18.8 & 4.1 & 0 \\
\hline Muinelo et al. ${ }^{18}$ & 2012 & España & 36 & 17.1 & 38.7 & 5.4 \\
\hline Kang et al. ${ }^{19}$ & 2011 & Korea & 52.5 & 4.5 & 40.9 & 2 \\
\hline Orhan et al. ${ }^{20}$ & 2011 & Turquía & 28.1 & 8.3 & 29.8 & 14.5 \\
\hline Videla et al. ${ }^{14}$ & 2010 & Chile & 25.64 & 5.13 & 42.31 & 11.54 \\
\hline Naitoh et al. ${ }^{15}$ & 2009 & Japón & 29.8 & 8.8 & 59.6 & 1.8 \\
\hline
\end{tabular}

BCDI: Bifurcación del conducto dentario inferior. 


\section{CONCLUSIÓN}

En conclusión se evidenció que la frecuencia de la BCDI fue del 10,75\% (161 casos). Se encontró mayor frecuencia en la quinta década de vida, no hubo diferencia estadísticamente significativa entre el sexo femenino y masculino ( $\mathrm{p}=0.49$ ). La distribución de la BCDI evidenció que el tipo Canal retromolar representó el 64\% (103 casos), seguido del tipo Canal sin confluencia anterior con $21.1 \%$ (34 casos) y minoritariamente el Canal dental y Canal con confluencia anterior con un $9.9 \%$ (16 casos) y 5\% (8 casos) respectivamente. No se encontraron casos del tipo canal bucal - lingual.

Conocer la frecuencia de la BCDI es importante debido al compromiso clínico de ésta variante anatómica que se pueda producir durante cualquier tratamiento odontológico, previniendo las consecuencias neurológicas que derivan de una lesión del nervio dentario inferior y de esta forma permitir al profesional realizar un adecuado procedimiento clínico evitando complicaciones que comprometan la salud del paciente.

\section{Correspondencia}

\section{Magaly Lizbet Quispe Huarcaya}

Correo electrónico: magaly.quispe.h@upch.pe

\section{REFERENCIAS BIBLIOGRÁFICAS}

1. Rouviére H, Delmas A. Anatomía humana descriptiva, topográfica y funcional. 11th ed. Delmas V, editor. Masson: Elsevier España; 2005.

2. Beltrán J, Abanto L, Meneses A. Disposición del conducto dentario inferior en el cuerpo mandibular: Estudio anatómico y tomográfico. Acta odontol venez. 2007; 45(3): 421-425.

3. Carter R, Keen E. The intramandibular course of the inferior alveolar nerve. J Anat. 1971; 108(3): 433-40.

4. Sanchis J, Peñarrocha M, Soler F. Bifid mandibular canal. J Oral Maxillofac Surg. 2003; 61(4):422-4.

5. Claeys V, Wackens G. Bifid mandibular canal: literature review and case report. Dentomaxillofac Radiol. 2005; 34(1):55-8.

6. Fukami K, Shiozaki K, Mishima A, Kuribayashi A, Hamada Y, Kobayashi K. Bifid mandibular canal: confirmation of limited cone beam ct findings by gross anatomical and histological investigation. Dentomaxillofac Radiol. 2012; 41(6):460-5.

7. Montaño J, Miranda M, Rojas M, Zuluaga C. Prevalencia de variables anatómicas en el recorrido de los conductos mandibulares. Estudio mediante tecnología cone beam. Facultad de odontología universidad de Antioquia. 2010; 22(1):23-32.

8. Sanchis J, Peñarrocha M, Soler F. Bifid mandibular canal. J Oral Maxillofac Surg. 2003; 61(4):422-4.

9. Nortje C, Farmen A, Grotepass F. Variations in the normal anatomy of the inferior dental (mandibular) canal: a retrospective study of panoramic radiographs from 3612 routine dental patients. Br J Oral Surg. 1977; 15(1):55-63.

10. Langlais R, Broadus R, Glass B. Bifid mandibular canals in panoramic radiographs. J Am Dent Assoc 1985; 110(6):923-6.

11. Schilling J, Schilling A, San Pedro J. Prevalencia de las bifurcaciones del canal mandibular, análisis en radiografías panorámicas digitales. Int J Odontostomat. 2010; 4(3):207-13.

12. Rossi P, Brucker M, Rockenbach M. Bifid mandibular Canals: panoramic radiographic analysis. Rev Cienc Med. 2009; 18(2):99-104.

13. Rouas P, Nancy J, Bar D. Identification of double mandibular canals: literature review and three case report with CT scans and cone beam CT. Dentomaxillofac Radiol. 2007; 36(1):34-8.

14. Videla J, Vergara M, Rudolph M, Guzmán C. Prevalencia de variables anatómicas en el recorrido de los conductos mandibulares. Estudio mediante tecnología Cone Beam. Rev Fac Odontol Univ Antioq. 2010; 22(1):23-32.

15. Naitoh M, Hiraiva Y, Aimiya H, Ariji E. Observation of bifid mandibular canal using cone beam computerized tomography. Int J Oral Maxillofac Implants. 2009; 24(1):155-9.

16. Kuribayashi A, Watanabe H, Imaizumi A, Tantanapornkul W, Katakami K, Kurabayashi T. Bifid mandibular canals: cone beam computed tomography evaluation. Dentomaxillofac Radiol. 2010; 39(4):235-9.

17. Rashsuren O, Choi J, Han W, Kim E. Assessment of bifid and trifid mandibular canals using cone-beam computed tomography. Imaging Sci Dent. 2014; 44(3):229-36.

18. Muinelo J, Suárez J, Fernández A, Marsillas S, Suárez M. Descriptive study of the bifid mandibular canals and retromolar foramina: cone beam CT vs panoramic radiography. Dentomacillofac Radiol. 2014; 43(5):1-8.

19. Kang J, Lee K, Oh M, et al. The incidence and configuration of the bifid mandibular canal in Koreans by using cone-beam computed tomography. Imaging Science in Dentistry. 2014;44:53-60.

20. Orhan K, Aksoy S, Bilecenoglu B, Sakul B, Paksoy C. Evaluation of bifid mandibular Canals with cone-beam computed tomography in a Turkish adult population: a retrospective study. Surg Radiol Anat. 2011; 33(6): 501-7. 